\title{
Editorial
}

Kari G. Smedstad MB FRCPC

\section{Obstetrical anaesthesia in Ontario}

thesia services, but $12 \%$ of these restricted its availability to Caesarean sections or to emergency situations only. In 1982, all the larger units, $83 \%$ of the intermediate (10-25 maternity beds), and $36 \%$ of the smaller units used epidural analgesia. In total, $68 \%$ of hospitals in Ontario had epidural experience in 1982, whereas $31 \%$ had never used epidurals. Almost $77 \%$ of hospitals with epidural experience used this technique for Caesarean sections by 1982. Lack of trained staff was the main reason for not providing regional anaesthesia for operative delivery in the other units.

By 1981 a cumulative total of 460,286 epidurals was administered in Ontario. Half of the hospitals allowed the nurses to perform "top-ups." Sixty-one percent of hospitals reported no life threatening problems related to epidural analgesia in labour. Most problems occurred in the remainder during the initiation of the epidural. Life threatening problems occurred in the absence of the anaesthetist in only two cases. In both, the anaesthetist was busy elsewhere in the hospital. One death from epidural anaesthesia was reported in Adamson's survey. In 1985 Crawford ${ }^{5}$ reported potentially life threatening complications after the initial dose to be $1: 4,500$, whereas after a "top-up" the ratio decreased to $1: 33,000$.

Oyston's survey tells us how obstetric anaesthesia services have changed in the last 12 yr. Overall, more hospitals now use epidurals, $75 \%$ vs $68 \%$. Epidurals are available $24 \mathrm{hr}$ a day in $29(20 \%)$ of Ontario's hospitals. That leaves 113 hospitals where epidurals are limited, used for special cases, or not at all. Nurses can give "top-ups" in $32 \%$ of hospitals in 1995 . Forty per cent of births in Ontario now take place in hospitals where comprehensive analgesia services are available $24 \mathrm{hr}$. Five per cent of hospitals in Ontario still perform all Caesarean sections under general anaesthesia. While Adamson's data are analyzed differently, the situation is not substantially different from 1983 . Oyston correctly stresses that every hospital should be able to provide regional anaesthesia for operative delivery. The trend now is towards spinal anaesthesia for elective Caesarean section, a technique that should be familiar to every anaesthetist.

The detailed information in Oyston's study is informative. Where epidurals are used, $90 \%$ of hospitals use
From the Department of Anaesthesia, McMaster University, 1200 Main St. West, Hamilton, Ontario L8N $3 Z 5$. 
continuous infusion pumps, and $70 \%$ add opioids to the infusate. Some use patient controlled analgesia, the survey does not state whether this is delivered epidurally or parenterally. Current literature attests to the safety of low dose infusion epidurals for pain relief in labour. ${ }^{6}$ The Canadian Anaesthetists' Society and the Ontario guidelines on epidural use may have persuaded practitioners to change their practice pattern in this regard. Patient controlled epidural analgesia is now feasible with modern infusion pumps, and has been shown to result in less drug delivery and greater patient satisfaction than epidural drugs infused on the orders of the anaesthetist. ${ }^{7}$ Use of intrathecal opioids in a combined epidural/spinal technique for labour is now gaining in popularity and is used in some teaching hospitals in Ontario.

What is the optimal epidural rate for labour analgesia? This question can only be answered by giving women a free choice of pain relief methods in labour. Only three hospitals in Ontario offered five methods of pain relief (opioids, transcutaneous nerve stimulation (TENS), nitrous oxide/oxygen, epidurals and patient controlled analgesia). When women are given a free choice, and not denied or "given" an epidural as a matter of course, the number who receive epidurals is remarkably similar in different centres. In our hospital, the rate hovers around $50-60 \%$. Ruderman ${ }^{8}$ reported that $76 \%$ of primiparae and $44 \%$ of multiparous women chose epidural analgesia at the Toronto hospital in 1988. Ong's survey of obstetrical anaesthesia at Winnipeg Women's Hospital ${ }^{9}$ showed an increase in epidural anaesthesia/analgesia from $28 \%$ in 1975 to $45 \%$ in 1983 . The use of inhalation techniques decreased from $40 \%$ to $4 \%$ in the same time frame. At the Flinders Medical Centre in Adelaide, South Australia, $45 \%$ of mothers receive epidural block for labour pain (Brownridge, personal communications). A survey of practice in Japan showed that $51 \%$ of institutions there use epidural analgesia as the preferred method of pain relief in the first stage of labour, however, the rate is not given. Lumbar epidural analgesia was the method of choice in France, where a rate of $70 \%$ is quoted. ${ }^{10}$

Oyston reports an overall epidural rate of $38 \%$, with $23 \%$ of Ontario hospitals having a rate of over $40 \%$. There is no argument with the fact that epidural analgesia is the most effective method of pain control during labour and child birth. Oyston presents data to support that epidurals are expensive as a means of relieving pain. Should cost dictate the use of the most effective pain relieving method for women in childbirth? The hidden cost of unrelieved pain for those who need epidurals must be considered. Since labour pain can cause stress resulting in metabolic acidemia, dysfunctional labour and fetal distress, and thus increased intervention, effective pain relief may save both money and lives in those parturients whose pain is intolerable and destructive. Also, since epidurals are safe and effective, is it ethical not to provide the choice of epidural analgesia to the majority of women giving birth in Ontario?

Oyston alludes to the ongoing controversy regarding the effect of epidural analgesia on the progress of labour. Most of the papers that found a detrimental effect of epidural analgesia are old and reflected epidural practices that are now outdated, or used questionable epidemiological methods. Recent publications have shown no such effects. ${ }^{11-13}$

The safety of epidural analgesia is well established, as Adamson, Oyston and others have pointed out. Brownridge advocates training midwives to administer "top-ups" and care for women with epidurals. ${ }^{14}$ Oyston's comments regarding midwives in Ontario are unnecessarily alarmist. The midwifery students in the Ontario schools are taught about pain and pain relief in labour, including care of epidurals. Epidural "top-ups" are a delegated medical act in Ontario, and any midwife or labour nurse who looks after patients with epidurals must have training and certification in the required skills.

The current survey looked at post operative analgesia after operative delivery. Post operative pain is often treated inadequately, and the obstetric situation is no different. It is well known that the "standard" intramuscular injection of meperidine is of little help. Patient controlled analgesia is expensive, as Oyston points out, and is often not needed for more than a day after surgery, but it is useful for women who have had a Caesarean section under general anaesthesia. With the move towards spinal anaesthesia for Caesarean section, a dose of an intrathecal opioid at the time of surgery, will provide good post operative analgesia for the majority of patients. One or two doses of intravenous analgesia, preferably with morphine, may be all that is needed before the patient can take oral medication.

The current survey has shown us that obstetrical analgesia and anaesthesia services in Ontario have improved somewhat in the last ten years. In 1993, the Ontario Medical Association wrote a position paper on perinatal services in the Province, documenting the current situation as precarious, particularly in smaller hospitals. ${ }^{15}$ The chapter on anaesthesia services pointed out that there are still hospitals in the province providing obstetrical services without access to an anaesthetist.

Should the recommendations from this report be acted upon, a new survey undertaken in another ten years may find that women in Ontario will be more satisfied with the choices available to them when they give birth. The document recommends that the hospitals "Facilitate the development of resources in order that all women can have a choice of pain relief modalities for childbirth. This 
includes the development of adequate training and maintenance of skills in analgesia and anaesthesia for practitioners providing the service."

\section{L'anesthésie obstétricale en Ontario}

Pour la femme, le soulagement de la douleur de l'accouchement sera toujours un sujet d'actualité. Plusieurs des livres et des magazines qu'elles lisent, traitent de méthodes efficaces et sans danger tant pour elles-mêmes que pour l'enfant. Toutefois, en face de la douleur obstétricale, le libre choix n'est pas encore une réalité au Canada. L'anesthésie obstétricale est devenue une sousspécialité, et dans les universités, on attache beaucoup d'importance à la recherche dirigée vers l'accouchement et la chirurgie qui l'entoure. Cependant, l'exercice de cette discipline de l'anesthésie n'est pas de qualité uniforme partout au pays. Il est donc très intéressant de prendre connaissance des résultats d'une nouvelle enquête sur l'anesthésie obstétricale en Ontario. ${ }^{1}$

In 1983, Adamson et al. réalisaient une première enquête sur tous les hôpitaux ontariens qui géraient des maternités. ${ }^{2}$ En Ontario, le premier service d'épidurales a vu le jour en 1956 et les autres hôpitaux, pour la plupart, ont suivi entre 1969 et 1970 . Au début des années 1980, cette politique avait semblé porter fruit car la mortalité maternelle associée à l'anesthésie avait chuté, ce qui fut attribué à l'introduction de l'anesthésie régionale. Avant 1970, on déplorait annuellement trois mortalités maternelles au moment de l'accouchement. Steinberg, ${ }^{3}$ dénombrait, de 1970 à 1974, quatre décès maternels en rapport avec l'anesthésie. En Colombie Britannique, la tendance était la même. ${ }^{4}$ Pendant la période 1955-1970, on a compté dix mortalités maternelles causés par l'anesthésie; de 1971 à 1986, un seul décès était causé par l'hyperthermie maligne.

Le Dr. Adamson s'inquiétait de la difficulté qu'auraient les hôpitaux communautaires de petite et de moyenne taille à dispenser ces services. Il s'est intéressé à l'accès à l'épidurale mais aussi à ces complications. Son étude portait sur 79\% de tous les lits de maternité de l'Ontario. En 1982, les petits hôpitaux n'offraient pas ces services et les hôpitaux intermédiaires ou majeurs refusaient de se lancer en anesthésie obstétricale. Tous les grands hôpitaux (35\% des hôpitaux relevés à ce moment) offraient des services d'anesthésie obstétricale de $24 \mathrm{hr}$, mais $12 \%$ de ces hôpitaux se limitaient aux césariennes et aux situations d'urgence. En 1982, tousles grands hôpitaux, $83 \%$ des intermédiaires (10 à 25 lits d'obstétrique) et $35 \%$ des petits hôpitaux offraient l'anesthésie épidurale. $\mathrm{Au}$ total, en Ontario, 68\% des hôpitaux avaient de l'expérience avec l'épidurale, alors que $31 \%$ n'en avait aucune. Presque 77\% des hôpitaux avec expérience offraient l'epidurale pour la césarienne en 1982. Ailleurs, l'absence d'un personnel formé constituait la raison principale pour ne pas proposer l'anesthésie pour les interventions obstétricales.

En 1991, les hôpitaux ontariens avaient administré un total cumulatif de 460,286 . La moitié des hôpitaux permettaient aux infirmières d'administrer les doses supplémentaires. Soixante et un pour cent des hôpitaux n'avaient pas rapporté de complications catastrophiques causées par l'épidurale. La plupart des problèmes étaient survenus au moment de la mise en marche de l'épidurale. Les problèmes catastrophiques sont survenus en l'absence des anesthésistes dans seulement deux cas. Dans chacun, l'anesthésiste était occupé ailleurs dans l'hôpital. Dans l'enquête d'Adamson, une seule mort a été attribué à l'épidurale. En 1985, Crawford ${ }^{5}$ rapportait des complications graves après la dose initiale dans un cas sur 4,400 , alors que cette proportion diminuait à 1 sur 33,000 après une dose supplémentaire.

L'enquête menée par Oyston nous révèle que l'anesthésie obstétricale a évolué au cours des 12 dernières années. En général, plus d'hôpitaux pratiquent l'épidural (75\% vs $68 \%$ ). L'épidurale est réalisable $24 \mathrm{~h}$ par jour dans $29(20 \%)$ des hôpitaux ontariens. Ce qui laisse 113 hôpitaux où l'épidurale est restreinte, réservée aux cas spéciaux ou n'est pas pratiquée. En Ontario, $40 \%$ des naissances se produisent dans des hôpitaux où des services complets d'anesthesie sont accessibles en tout temps. Dans cinq pour cent des hôpitaux ontariens, on continue de réaliser la césarienne sous anesthésie générale. Même si les données d'Adamson sont analysées différemment, la situation actuelle n'est pas tellement différente de celle de 1983. Oyston insiste avec raison sur le fait que tous les hôpitaux devraient procurer l'anesthésie régionale pour la chirurgie obstétricale. La tendance actuelle favorise la rachianesthésie pour la césarienne, et tous les anesthésistes sont familiers avec cette technique.

On a beaucoup à apprendre de l'enquête d'Oyston. Là où l'épidurale est offerte, $90 \%$ des hôpitaux utilisent la pompe à perfusion, et $70 \%$ ajoutent de la morphine à la solution perfusée. Quelques hôpitaux offrent l'anesthésie autocontrôlée, mais l'étude ne précise pas si c'est par la voie épidurale ou la voie intraveineuse. Les études les plus récentes confirme la sécurité des perfusions épidurales à faibles doses administrées pour soulager la douleur pendant du travail. ${ }^{6}$ Les directives sur l'épidurale pro- 
mulgues par la Société Canadienne des Anesthésistes et par celle de l'Ontario ont persuadé les anesthésistes de changer leur attitude. Grâce aux pompes à perfusion modernes, l'épidurale autocontrôlée est réalisable; en outre, il a été démontré que la quantité d'analgésique administrée était moindre, et que le degré de satisfaction était plus élevé que lorsque les agents étaient administré de façon intermittente. ${ }^{7}$ L'utilisation des morphinique sousarachnoïdiens par rachianesthésie-épidurale combinées est de plus en plus populaire, et, en Ontario, on lutilise surtout dans les hôpitaux d'enseignement.

Quel est le pourcentage d'anesthésies épidurales souhaitable pour l'analgésie pendant travail? Cette question ne peut être résolue qu'en accordant aux parturientes le libre choix de la méthode qu'elles désirent. Seulement trois hôpitaux ontariens offrent le choix entre cinq méthodes (morphiniques, stimulation électrique transcutanée (TENS), protoxyde d'azote/oxygène, épidurale et anesthésie autocontrôlée). Quand on offre un choix absolu aux parturientes et qu'on ne leur refuse ni leur administre contre leur gré l'épidurale, le nombre de celles qui acceptent l'épidurale n'est pas tellement différent entre les institutions. Dans notre hôpital, le pourcentage varie entre 50 et $60 \%$. Ruderman ${ }^{8}$ rapporte qu'en 1988 , à l'hôpital de Toronto, $76 \%$ des primipares et $44 \%$ des multipares ont choisi une analgésie épidurale. L'enquête d'Ong au Winnipeg Women's Hospital ${ }^{9}$ révèle une augmentation de $28 \%$ en 1975 a $45 \%$ en 1993 . L'anesthésie par inhalation a baissé de $40 \%$ à $4 \%$ pendant la même période. En Australie, au Flinders Medical Centre d'Adelaïde, $45 \%$ des parturientes acceptent l'épidurale pour le travail (Brownridge, communication personnelle). Au Japon, un sondage a montré que dans $51 \%$ des institutions, lanalgésie épidurale etait utilisée comme principale méthode pour l'allégement de la douleur pendant le premier stage du travail; le pourcentage des institution n'est toutefois pas mentionné. En France, où le pourcentage est de 70\%, l'analgésie épidurale lombaire constitue la méthode de choix. ${ }^{10}$

Oyston rapporte que $38 \%$ des hôpitaux ontariens dont $23 \%$ ont un taux de $40 \%$ et plus, pratiquent l'épidurale obstétricale. Il est universellement reconnu que l'analgésie épidurale est la méthode la plus efficace pour le contrôle de la douleur pendant le travail et l'accouchement. Son utilisation devrait-elle être limitée par son coût? A cet égard, les coûts obscurs de la douleur non soulagée doivent être pris en considération. Comme la douleur du travail cause un stress qui provoque de l'acidose métabolique, de la dystocie et de la détresse foetale, et augmente ainsi le nombre des interventions, le soulagement de la douleur du travail peut à la fois sauver de l'argent et des vies lorsque la douleur devient intolérable et nuisible. En outre, comme l'analgésie épidurale est sûre et efficace, est-ce moral de ne pas l'offrir à la majorité des femmes lorsqu'elles accouchent.

Oyston mentionne aussi la controverse qui fait continue de faire rage au sujet de la répercussion de l'épidurale sur la progression du travail. La plupart des articles qui décrivent cet aspect nocif de l'analgésie épidurale sont d'un autre âge et concernent une pratique démodée de l'épidurale, ou s'appuient sur des méthodes épidémiologiques douteuses. Les publications plus récentes ne révèlent pas cet inconvénient. ${ }^{11-13}$

La sécurité de l'analgésie épidurale est bien établie, comme le soulignent Adamson, Oyston et d'autres. Brownridge préconise la formation de sages-femmes pour administrer les doses supplémentaires et les soins nécessaires aux parturientes sous épidurale. ${ }^{14}$ Les commentaires d'Oyston sur les sages-femmes en Ontario sont sans raison alarmistes. En Ontario, on renseigne les étudiantes sages-femmes sur la douleur et son soulagement, dont la surveillance des épidurales. En Ontario, la dose supplémentaire est un acte médical délégué, et toute sagefemme ou infirmière oeuvrant en maternité est formée et confirmée pour cette activité.

L'enquête actuelle sintéresse aussi à l'anesthésie postopératoire après la chirurgie obstétricale. La douleur postopératoire est souvent traitée insuffisamment et ce n'est pas différent en obstétrique. Il est bien connu que l'injection traditionnelle de mépéridine intramusculaire n'est d'aucun secours. L'analgésie autocontrôlée coûte cher, comme le fait remarquer Oyston, et son utilité ne dépasse pas une journée après la chirurgie; elle est toutefois plus utile après les césariennes sous anesthésie générale. Avec l'évolution actuelle qui favorise la rachianesthésie pour la césarienne, l'ajout d'une dose sous-arachnoïdienne de morphinique au moment de linduction, procurera une bonne analgésie postopératoire pour la majorité des patientes. Une ou deux doses intraveineuses d'analgésique, tout au plus, preférablement de la morphine, suffiront en attendant que la patiente puisse tolérer une médication orale.

L'enquète actuelle nous montre, qu'en Ontario, l'analgésie et l'anesthésie obstétricales se sont en fin de compte améliorées. En 1993, l'Association Médicale de l'Ontario a statué sa position sur les services périnataux provinciaux, avec la mention qu'il étaient précaires, et cela, surtout dans les petits hôpitaux..$^{15}$ Le chapitre concernant l'anesthésie obstétricale soulignait qu'il existait des services d'obstétrique où la disponibilité d'anesthésistes était nulle.

Si les recommendations de cette enquête étaient entérinées, une nouvelle enquête entreprise dans dix ans pourrait montrer que les parturientes de l'Ontario sont plus satisfaites des choix qui leur sont offerts à l'accouchement. Le document recommande que les hôpitaux 
« facilitent le développement des ressources, de sorte que toutes les femmes puissent avoir le choix entre différentes méthodes. Ceci inclut la mise en oeuvre d'une formation adéquate et le maintien de la compétence en analgésie et en anesthésie pour les praticiens qui procurent les services ").

\section{References}

1 Oyston J. Obstetrical anaesthesia in Ontario. Can J Anaesth 1995; 42: 1117-25.

2 Adamson DH, Burton HJ, Heidenheim P. Epidural analgesia in obstetrics: the Ontario experience. Final Report. Survey conducted by the Faculty of Nursing and the Health Care Research Unit, Department of Anaesthesia, The University of Western Ontario, London, Ontario. March 1993; 1-64.

3 Steinberg $W M$, Farine $D$. Maternal mortality in Ontario from 1970 to 1980. Obstet Gynecol 1985; 66: 510-2.

4 Wittmann BK, Murphy KJ, King JF, Yuen BH, Shaw D, Wittmann $A G$. Maternal mortality in British Columbia in 1971-86. Can Med Assoc J 1988; 139: 37-40.

5 Crawford JS. Some maternal complications of epidural analgesia for labour. Anaesthesia 1985; 40: 1219-25.

6 Shnider SM, Levinson G, Ralson DH. Regional anesthesia for labor and delivery. In: Shnider SM, Levinson G (Eds.). Anesthesia for Obstetrics, 3rd ed. Maryland: Williams \& Wilkins, 1993: 135-53.

7 Gambling DR, Huber CJ, Berkowitz J, et al. Patientcontrolled epidural analgesia in labor: varying bolus dose and lockout interval. Can J Anaesth 1993; 40: 211-7.

8 Ruderman $J$, Carroll JC, Reid AJ, Murray MA. Are physicians changing the way they practise obstetrics? Can Med Assoc J 1993; 148: 409-15.

9 Ong B, Cohen MM, Cumming M, Palahniuk RJ. Obstetrical anaesthesia at Winnipeg Women's Hospital 1975-83: anaesthetic techniques and complications. Can J Anaesth 1987; 34: 294-9.

10 Arai $M$, Nishijima $M$, Tatsumi.H. Analgesia and anesthesia during labor in Japan and developed countries. Asia Oceania J Obstet Gynaecol 1989; 15: 213-21.

11 Dewan DM, Cohen SE. Epidural analgesia and the incidence of cesarean section. Time for a closer look (Editorial). Anesthesiology 1994; 80: 1189-92.

12 Chestnut DH, McGrath JM, Vincent RD Jr, et al. Does early administration of epidural analgesia affect obstetric outcome in nulliparous women who are in spontaneous labo? Anesthesiology 1994; 80: 1201-8.

13 Chestnut DH, Vincent RD Jr, McGrath JM, Choi WW, Bates JN. Does early administration of epidural analgesia affect obstetric outcome in nulliparous women who are receiving intravenous oxytocin? Anesthesiology 1994; 80: 1193-200.

14 Brownridge $P$. Epidural medication after the initial dose: reflections on current methods of administration during labour. Anaesth Intensive Care 1990; 18: 300-8.

15 Smedstad KG. Obstetrical Anaesthesia Services in Small Hospitals. In: Trends in Reproductive Care: A Medical Perspective. Committee on Reproductive Care of the Ontario Medical Association, Toronto, Canada. 1992: 25-31. 